\title{
Operational space weather service for GNSS precise positioning
}

\author{
N. Jakowski, S. M. Stankov, and D. Klaehn \\ German Aerospace Centre (DLR), Institute of Communications and Navigation, Kalkhorstweg 53, 17235 Neustrelitz, \\ Germany
}

Received: 22 February 2005 - Revised: 18 June 2005 - Accepted: 20 July 2005 - Published: 22 November 2005

Part of Special Issue "1st European Space Weather Week (ESWW)"

\begin{abstract}
The ionospheric plasma can significantly influence the propagation of radio waves and the ionospheric disturbances are capable of causing range errors, rapid phase and amplitude fluctuations (radio scintillations) of satellite signals that may lead to degradation of the system performance, its accuracy and reliability. The cause of such disturbances should be sought in the processes originating in the Sun. Numerous studies on these phenomena have been already carried out at a broad international level, in order to measure/estimate these space weather induced effects, to forecast them, and to understand and mitigate their impact on present-day technological systems.

SWIPPA (Space Weather Impact on Precise Positioning Applications) is a pilot project jointly supported by the German Aerospace Centre (DLR) and the European Space Agency (ESA). The project aims at establishing, operating, and evaluating a specific space-weather monitoring service that can possibly lead to improving current positioning applications based on Global Navigation Satellite Systems (GNSS). This space weather service provides GNSS users with essential expert information delivered in the form of several products - maps of TEC values, TEC spatial and temporal gradients, alerts for ongoing/oncoming ionosphere disturbances, etc.
\end{abstract}

Keywords. Ionosphere (Ionospheric disturbances; Modeling and forecasting; Instruments and techniques)

\section{Introduction}

The Space Weather is defined as the set of all conditions - on the Sun, and in the solar wind, magnetosphere, ionosphere and thermosphere - that can influence the performance and reliability of space-borne and ground-based technological systems and can endanger human life. Several studies have already been performed showing clear evidences of space

Correspondence to: S. M. Stankov

(stanimir.stankov@dlr.de) weather - induced adverse effects on the Earth's ionosphereplasmasphere system (Jakowski et al., 1998, 1999, 2002a, 2002b, 2003a, 2004b; Langley, 2000; Makela et al., 2001; Stankov, 2002; Skone and Shrestha, 2002). Such effects can ultimately cause various types of problems including: range errors, rapid phase and amplitude fluctuations (radio scintillations) of satellite signals, etc., that may lead to pronounced signal degradation, degradation in the system performance, its accuracy and reliability (Moulsley and Vilar, 1982). Also reported are strong voltage fluctuations and harmonic frequencies in the electricity, electrical power line shutdowns and blackouts (Harang, 1941; Kappenman, 1996; Boteler and Jansen Van Beek, 1999). Being electrical conductors, oil and gas pipelines are also vulnerable to strong geomagnetic disturbances (Pirjola et al., 1999); geomagnetically induced currents (GICs) produce additional voltages between the pipeline and the ground which can easily exceed the protective voltage, therefore damaging the corrosion protection. In addition, GICs also may hamper rail traffic by disturbing some types of signalling systems. Aviation and space flights are particularly at risk due to increased radiation and possible navigation/telecommunication problems (Oksanen, 1998; Koskinen et al., 1999). Apart from the increased risk of using the affected systems, the negative impact of adverse space weather can also have a purely economic dimension. With the future advancement of technology, the abovementioned risks and possible financial losses will certainly increase unless suitable protective measures are taken in advance.

Since trans-ionospheric propagation effects are responsible for positioning errors in satellite-based navigation and positioning, the GNSS users need to apply appropriate mitigation techniques, such as corrections by the use of: dual frequency techniques, model corrections, local or global augmentation systems. Strong gradients in the horizontal TEC structure and small-scale structures of the ionospheric plasma may seriously complicate or even prevent the resolution of phase ambiguities in precise geodetic and surveying networks. Principally, in reference networks, the ionospheric 

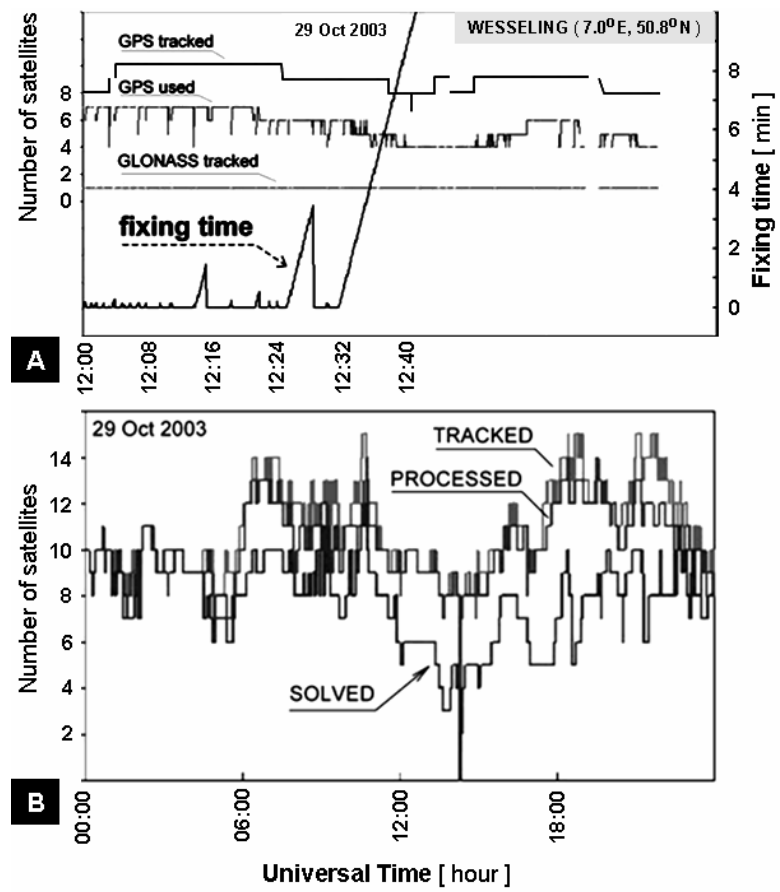

Fig. 1. A: Time required to fix ambiguities: a case during the ionospheric storm on 29 October 2003. Notice the increased fixing time (more than $3 \mathrm{~min}$ ) at 12:24 UT and the breakdown at 12:32 UT. B: Storm effects demonstrated on the number of tracked, processed, and solved satellites. Normally, the number of processed satellites should be equal to the number of solved satellites.

corrections degrade when the spatial de-correlation of propagation terms increases, particularly in the course of severe ionospheric storms. Whereas medium-scale variations in time and space, such as Traveling Ionospheric Disturbances (TIDs), mainly impact reference networks, local, small-scale irregularities may cause radio scintillations, inducing severe signal degradation and even loss of lock. Amplitude scintillations and phase fluctuations are caused by refractive and diffractive scatter by ionospheric plasma-density irregularities, especially at equatorial and auroral-to-polar latitudes.

SWIPPA (Space Weather Impact on Precise Positioning Applications) is a project aiming at establishing, operating, and evaluating a specific space-weather monitoring service that can possibly lead to improving current positioning applications based on GNSS (Jakowski et al., 2002c, 2003b, 2004a). The SWIPPA service centre operates a powerful data processing system which, working in both real-time and post-processing modes, provides the project consortium members with essential expert information delivered in the form of several products - maps of TEC values, TEC spatial and temporal gradients, alerts for ongoing/oncoming ionosphere disturbances, etc.

Presented in this paper are several case studies of problems arising from the space weather impact on GNSS-based applications and the SWIPPA operational service which can be used to help the GNSS users to mitigate the above mentioned problems.

\section{Space weather effects observed on GNSS applications}

There are several problems currently experienced by GNSS users which can be related to space weather effects.

\subsection{Increased time required to fix GNSS signal phase am- biguities}

GNSS reference networks provide ionospheric corrections to the users in order to help them achieve centimetre level accuracy. A very important factor determining the performance of such a GNSS reference network service is the time required to solve the phase ambiguities. The strong variability of the ionosphere obviously affects the determination of phase ambiguities. Presented here is a case when such difficulties arise, and at a certain stage it becomes even impossible to fix the ambiguities. A typical example of such a problem occurred on the geomagnetically disturbed day of 29 October 2003 at 12:32 UT: the ambiguity fixing time extended so much that it became impossible to achieve a fixed solution (Fig. 1A). During such periods it is very difficult to model the error influences and therefore the user cannot obtain precise positioning. However, nowcast and/or forecast of the ionospheric disturbances can certainly help the GNSS users, at least to be aware of such disturbances and to recognize that a problem is (to be) expected.

\subsection{Discrepancies between "processed" and "solved" GNSS satellites}

Specialised positioning algorithms are used for "solving" the phase ambiguities. In order to maintain a regular and reliable service, it is necessary to ensure that, at any given moment in time, the signal phase ambiguities are "solved" for at least 5 GNSS satellites. To ensure this minimum number of "solved" satellites, each particular ground receiving station tracks as many GPS and GLONASS satellites as possible. Some of these "tracked" satellites cannot be used due to restrictions imposed on the satellite elevation angle (elevation cutoff criterium), the signal-to-noise ratio (SNR criterion), etc. The remaining satellites are being "processed", i.e. their signals are used in the processing software for fixing the ambiguities. Criteria also exist when trying to solve the ambiguities, for example, the errors should be small and the solution stable. Therefore, due to the "solving criteria" some of the "processed" satellites cannot be used. Thus, the satellites for which the ambiguities have been fixed (solved), are called "solved" satellites and their number is equal to or less than the number of processed satellites. Under normal conditions, the number of "processed" satellites should equal the number of "solved" satellites. This is exactly the situation between 00:00 UT and 06:00 UT on 29 October 2003 when the numbers were sometimes between 11 and 6 satellites (Fig 1B). Obvious discrepancies between processed and solved satellites started to occur shortly after the first geomagnetic storm started at 06:00UT. The situation has gradually worsened as the storm developed and the solved/processed percentage 
ratio dropped to only $20-30 \%$. Moreover, the number of solved satellites fell even below the critical level, meaning that the positioning was no longer reliable. The worst happened at around 14:00 UT when the number of solved satellites dropped to the zero level and the service effectively stopped. Such extreme situations are rare, but should they occur, they could seriously disturb GNSS-based applications. As the storm entered the recovery phase (at around 15:00 UT), the situation slightly improved - the number of solved satellites increased above the threshold level. However, the discrepancies were still large and the danger of going back to unreliability was present until 22:00 UT on 29 October 2003.

\subsection{Phase and amplitude fluctuations}

Phase and amplitude fluctuations are caused by the refractive and diffractive scattering due to ionospheric plasma irregularities, especially at equatorial and polar latitudes (Beniguel, 2002; Beniguel et al., 2004). The problem with such smallscale irregularities is that they can lead to cycle slips and tracking losses (scintillations). Medium-scale irregularities (TIDs) and strong ionospheric gradients may cause different signals at the reference station and the user site, i.e. the correction message transmitted from the GNSS reference network centre to the user in the field will be definitely wrong. The strong TEC dynamics at high latitudes, observed during the storm on 6 April 2000, were of the order of $40 \times 10^{16} \mathrm{~m}^{-2}$ $(\approx 6.50 \mathrm{~m}$ on the GPS L1 frequency) which caused difficulties for the precise positioning and navigation (Jakowski et al., 2002b). During this event, rapid phase fluctuations have been detected by both the GPS and GLONASS receivers in mid-latitude Europe (Fig. 2A).

\subsection{Decreased reference network model integrity}

The Network Model Integrity (NMI) is an important operational parameter used in the GNSS reference network software to control both the integrity and the quality of the satellite positioning services. In fact, the NMI module is used to describe the potential non linear residual errors in the generated data transmitted to the user. The non linear error is estimated in the following manner. Initially, the ionospheric influence on GNSS signals is determined and then the linear parts of these effects are removed by applying ionospheric and geometric corrections to the raw data. The influence on the user position is interpolated from the influence determined on the surrounding reference stations. During periods of disturbed ionosphere, however, the ionospheric residuals cannot be considered linear, even locally. The nonlinear error is determined by omitting one station from the interpolation, comparing the interpolation results for all satellites at that station with the real measurements, then computing the weighted RMS over all satellites at one epoch, and accumulating these weighted RMS over one hour to obtain a 95\% distribution. As mentioned, if the residual error is large, the user may experience longer times for fixing the ambiguities
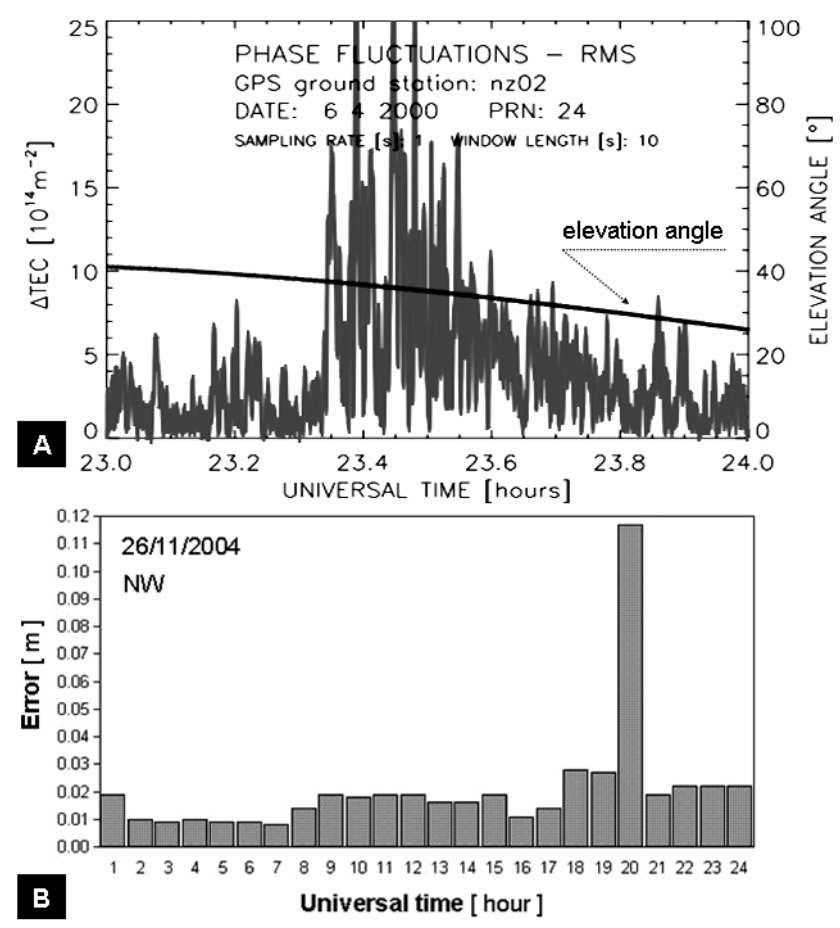

Fig. 2. A: Phase fluctuations observed during the 6-7 April 2000 storm. B: Network Model Integrity monitoring. Increased errors observed from 19:00 UT to 20:00 UT on 26 November 2004 in the northwest German reference sub-network.

and increased inaccuracies. An NMI plot (Fig. 2B) shows the averaged error over an entire reference network (or if the network is too large - over a sub-network), i.e. the average of the errors at the network stations. The horizontal axis shows the hour of the day (universal time), while the vertical axis shows the error. Recent experiences teach that it is hardly possible to obtain precise and accurate results while the error is larger than $8 \mathrm{~cm}$. If the error is between 4 and $8 \mathrm{~cm}$, the reference network user has to accept longer times to fix ambiguities. Smaller values represent a quiet ionosphere.

\section{Case studies}

Reported here are adverse space weather effects on precise positioning, observed during two recent strong ionospheric storms, on 29 October 2003 and on 25 July 2004. The analysis is focused on the ionosphere perturbations' impact on positioning - the increased time required to fix GNSS signal phase ambiguities; discrepancies between the numbers of tracked, processed and solved GNSS satellites; strong phase and amplitude fluctuations, etc. Also detailed are the observations of the geophysical conditions during these two storms and the means to mitigate the adverse effects. On 29 October 2003, strong large-scale ionospheric perturbations that can potentially lead to performance degradation of reference networks were first detected in the northern polar region (Fig. 3). When the solar wind energy, measured by the Northern Hemispheric power index (Foster et al., 1986), 

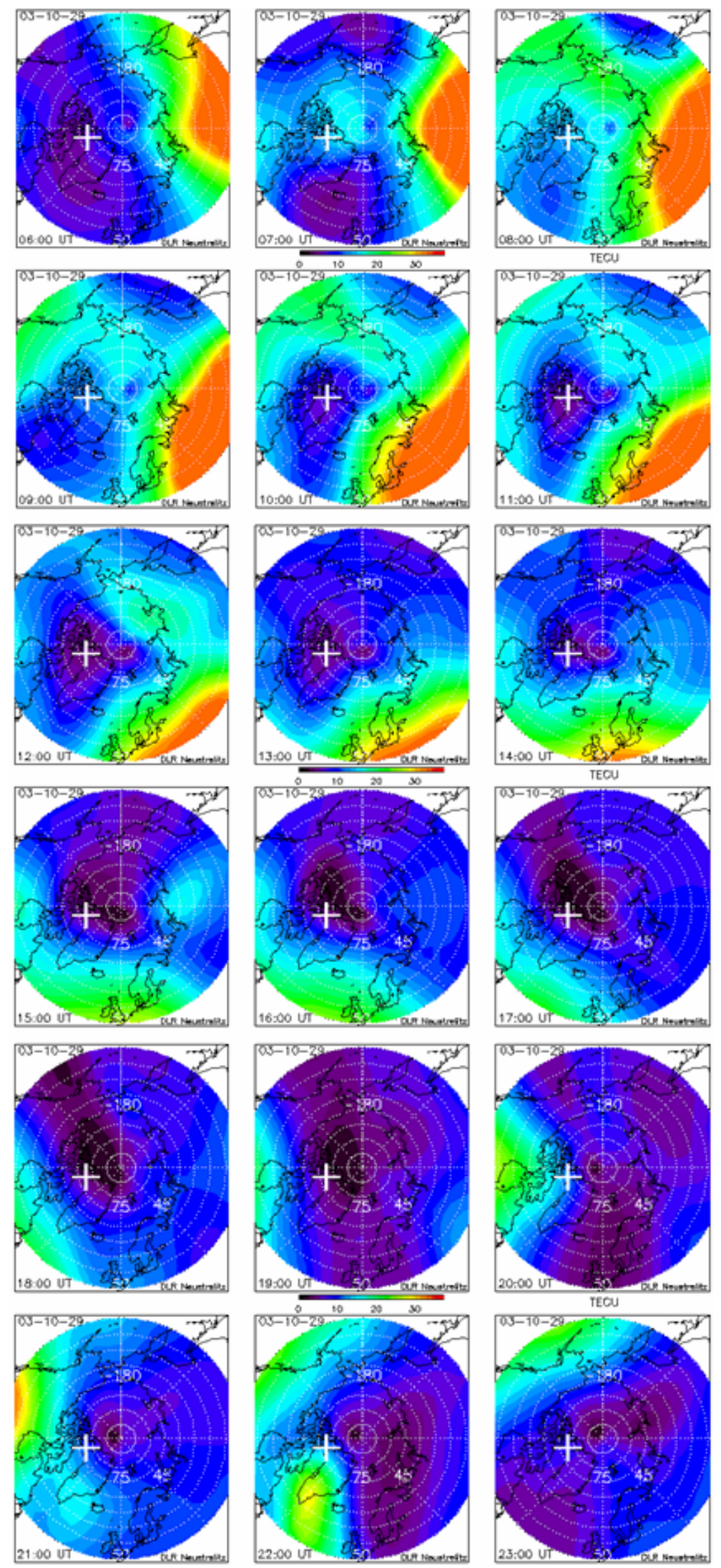

Fig. 3. Ground-based GPS TEC observations over the North Pole region in the 06:00 UT-23:00 UT period on 29 October 2003. 
couples with the magnetosphere, strong convection electric fields are generated that may be accompanied by direct particle precipitation producing additional ionization in the bottom-side ionosphere. The action of such a strong convection electric field is visible in the polar TEC maps from 07:00-12:00 UT when a significant horizontal plasma transport from the dayside towards the nightside ionosphere took place, probably via the electromagnetic $\boldsymbol{E} \times \boldsymbol{B}$ drift. A second event of enhanced perturbation activity is seen in the polar TEC maps around 21:00 and 22:00 UT; however, the induced perturbations are more significant over the South Pole (Stankov and Jakowski, 2004). These and other observations clearly indicate that the polar ionosphere is a kind of "space weather kitchen", where perturbation processes are generated and then propagate towards lower latitudes (Foerster and Jakowski, 2000; Jakowski et al., 2003a). Unfortunately, not much is known so far about such propagation processes. A careful analysis of these observations will contribute to improving our understanding of large-scale perturbation processes and their propagation towards lower latitudes (Stankov et al., 2005). Thus, the permanent observations also offer the unique chance of developing techniques/procedures that can be applied for predicting severe ionospheric perturbations expected to occur even at middle latitudes with a certain time delay (Stankov et al., 2001).

The last 10 days of July 2004 were remarkably active considering the approaching minimum in the current solar activity cycle. Three consecutive major storm events occurred, with the $D_{s t}$ index reaching $-104 \mathrm{nT}$ on $23 \mathrm{July},-150 \mathrm{nT}$ on 25 July and $-167 \mathrm{nT}$ on 27 July. The IMF and geomagnetic fields were seriously disturbed. Again, several problems were reported by GNSS users but here we will take a closer look at the reference network model integrity only. Presented here are the results of the network model integrity tool for 25 July 2004 (Fig. 4). The plots of the different subnets are shown according to the area they cover in Germany (NW - North West, NE - North East, MW - Mid West, ME - Mid East, SW - South West, SE - South East). Around noon, higher-than-usual values were seen in the eastern areas. Later, the influence was detected in the western areas, as well.

\section{Operational service for precise positioning applica- tions of GNSS}

First motivated by several problems experienced by GNSS users, then by analysing these problems and considering the research and operational monitoring experience, the DLR team initiated a project SWIPPA (Space Weather Impact on Precise Positioning Applications), aiming at establishing and operating a specific space-weather monitoring service for improving current Global Navigation Satellite System (GNSS) applications (http://www.kn.nz.dlr.de/swippa/index. $\mathrm{htm})$. Within this project, real-time data products and services (TEC maps, TEC spatial and temporal gradients maps, cycle slips number, geophysical conditions warnings, etc.)

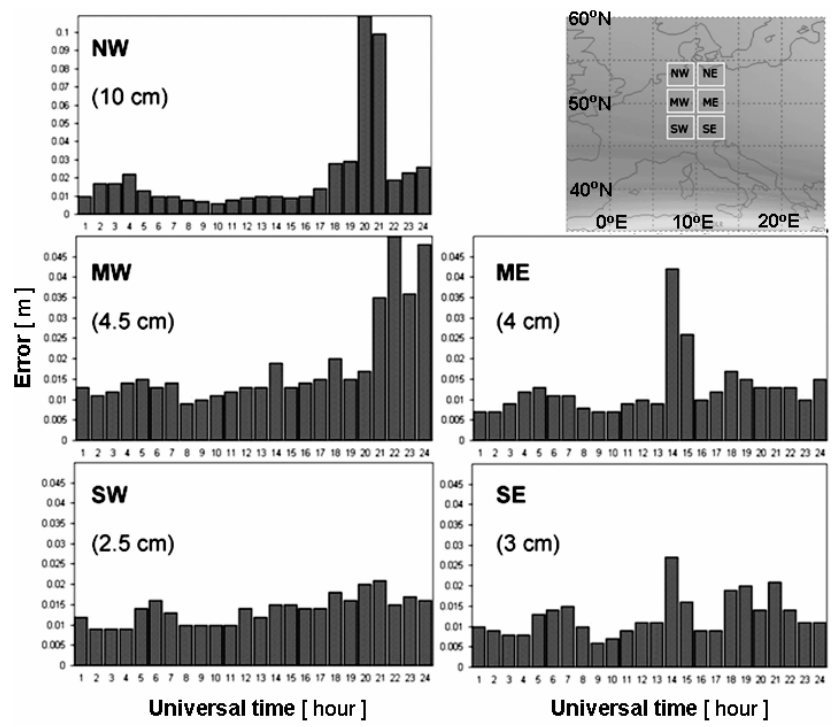

Fig. 4. Adverse space weather effects on the operational quality of reference networks. The network model integrity on 25 July 2004 for the NW, MW, SW, ME, SE areas of Germany (cf. the TEC map in the upper right corner).

are offered to the consortium members, designated users, and the general public. These products, based on the information of the actual and predicted state of the ionosphereplasmasphere system, provide only the type of space weather information which GNSS users really need for the execution of their day-to-day tasks.

\subsection{Processing system}

The SWIPPA service centre operates a powerful data processing system (Fig. 5), working in both real-time and postprocessing modes, to provide the customers with actual information and to feed the forecasting module with the newest information available from our partners, from the Space Weather European Network (SWENET) community, and from external sources, like world data centres. The SWIPPA data processing system generates near real-time products based on data from the ascos (http://ascos.ruhrgas.de/) and the SAPOS (http://www.sapos.de/) reference networks. Typically, these networks are operated at monitoring stations, with a sampling rate of 1 measurement per second $(1 \mathrm{~Hz}$ sampling rate). Depending on the data source, the data is formatted and transferred to the SWIPPA processing facility at DLR, using the in-house data format and data transfer protocols. According to the types of products being generated, several processing chains had to be designed and implemented. Because of the experimental nature of the project, aimed at demonstrating the potential of the space weather information as derived from ionospheric monitoring systems, we have taken advantage of some pre-existing DLR software components and universal processing structures that have been applied by DLR in several projects before. 


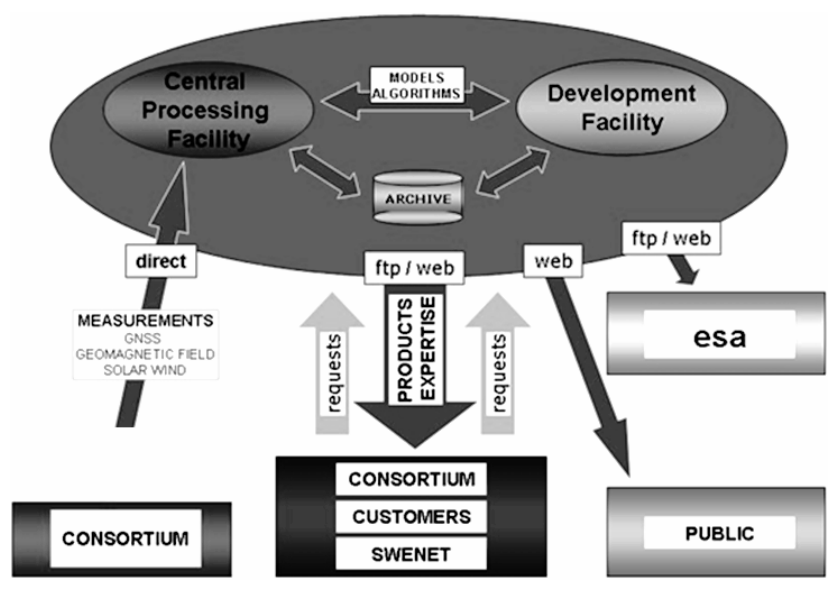

Fig. 5. A schematic view of the SWIPPA operational system.

\subsection{Products and services}

Following the SWIPPA consortium user requirements, several products were developed and expert services established.

\subsubsection{TEC mapping}

The GPS data allow for the determination of slant TEC values along numerous satellite-receiver links over the European area. The instrumental biases are separated from the observations by assuming a second-order polynomial approximation for TEC variations over the observing GPS ground station. Both the TEC and the instrumental satellite-receiver biases are estimated simultaneously by a Kalman filter run over $24 \mathrm{~h}$. Thus calibrated, the slant TEC data are mapped to the vertical by applying a mapping function which is based on a single layer approximation at height of $400 \mathrm{~km}$. Finally, the observed TEC data are combined with a regional TEC model, the Neustrelitz TEC Model (NTCM), in a way that the resulting map (Fig. 6A) provides measured values at some locations and model values at regions without measurements. The advantage of such an assimilation technique is that even in the case of a low number of measurements, it delivers reasonable ionospheric corrections which can be provided to the users to help them enhance accuracy and integrity of positioning (Jakowski, 1996; Jakowski et al., 1996).

\subsubsection{TEC gradients mapping}

The observance of strong ionospheric gradients indicates the development of highly dynamic processes in the Earth's ionosphere-plasmasphere system, with the potential of having degrading effects on positioning/navigation. For example, both WAAS (Wide Area Augmentation System) and LAAS (Local Area Augmentation System) receivers utilize carrier-smoothing filters to reduce the effects of multipath and thermal noise at the aircraft. By applying such filters, users can ultimately improve accuracy. However, the presence of significant ionospheric gradients can introduce a bias into this filter's output; if unmitigated, this bias can grow to be much larger than the noise and multipath effects that the filter is supposed to reduce. Ionospheric gradients are reportedly capable of inducing biases as large as $20-30 \mathrm{~m}$ (Walter et al., 2004). The production of TEC gradients maps is based on the calculation of temporal and spatial gradient at each grid point in the European region (optimally, $1 \mathrm{deg}$ spatial resolution). Three types of gradient maps are created - temporal (time) gradients (Fig. 6B), latitudinal gradients (Fig. 6C), and longitudinal gradients (Fig. 6D).

\subsubsection{Geophysical/space weather condition warning}

After analyzing and synthesizing the incoming geophysical information, short messages are forwarded to users, warning them about on-going ionosphere disturbances. The geophysical warning (Fig. 7) is based mostly on the geomagnetic index $K_{p}$ estimations/predictions using solar wind observations (wind density, speed) on board the satellite ACE (Advanced Composition Explorer). A neural network algorithm (Costello, 1997), trained on the response of $K_{p}$ to solar wind parameters, uses most recent solar wind measurements to predict $K_{p}$. Additional real-time data, such as the geomagnetic field's horizontal component (Niemegk: $12.7^{\circ} \mathrm{E}$, $\left.52.1^{\circ} \mathrm{N}\right)$ and the percentage deviation of the ionospheric critical frequency $(f o \mathrm{~F} 2)$ from monthly medians (Juliusruh: $13.4^{\circ} \mathrm{E}, 54.6^{\circ} \mathrm{N}$ ), are also considered but as validation tools rather than values on which to base the warning. The warning message is kept simple, so it can be conveniently used by programmers via the ftp protocol.

\subsection{Exemplary results}

As an example, demonstrated here is how SWIPPA products and services can really help. By generating high-resolution maps of the TEC spatial and temporal gradients, the sharp increase in TEC in the northeast region during the 25 July 2004 storm becomes quite obvious (Figs. 6E and F) and its propagation in the southward and westward direction can easily be traced (Figs. 6G and H). As the front of the detected disturbances moves through Europe, it causes various problems, including the decrease in the network model integrity (Fig. 4). What is important to mention here is that, in this case, probably due to the exhaustion of the ionosphere during the persisting storm conditions from the previous days, largescale TEC maps of the Polar and European regions may not provide clear and early indications for the developing storm conditions at lower latitudes. Such clear and advanced indications have been successfully delivered by the new, direct, high-resolution, and frequent mapping of the TEC values and TEC spatial/temporal gradients.

\section{Summary and outlook}

Several cases were presented of adverse space-weather effects on precise positioning, observed during the ionospheric storms on 29 October 2003 and 25 July 2004. The focus was on the increased fixing time, the discrepancies between 

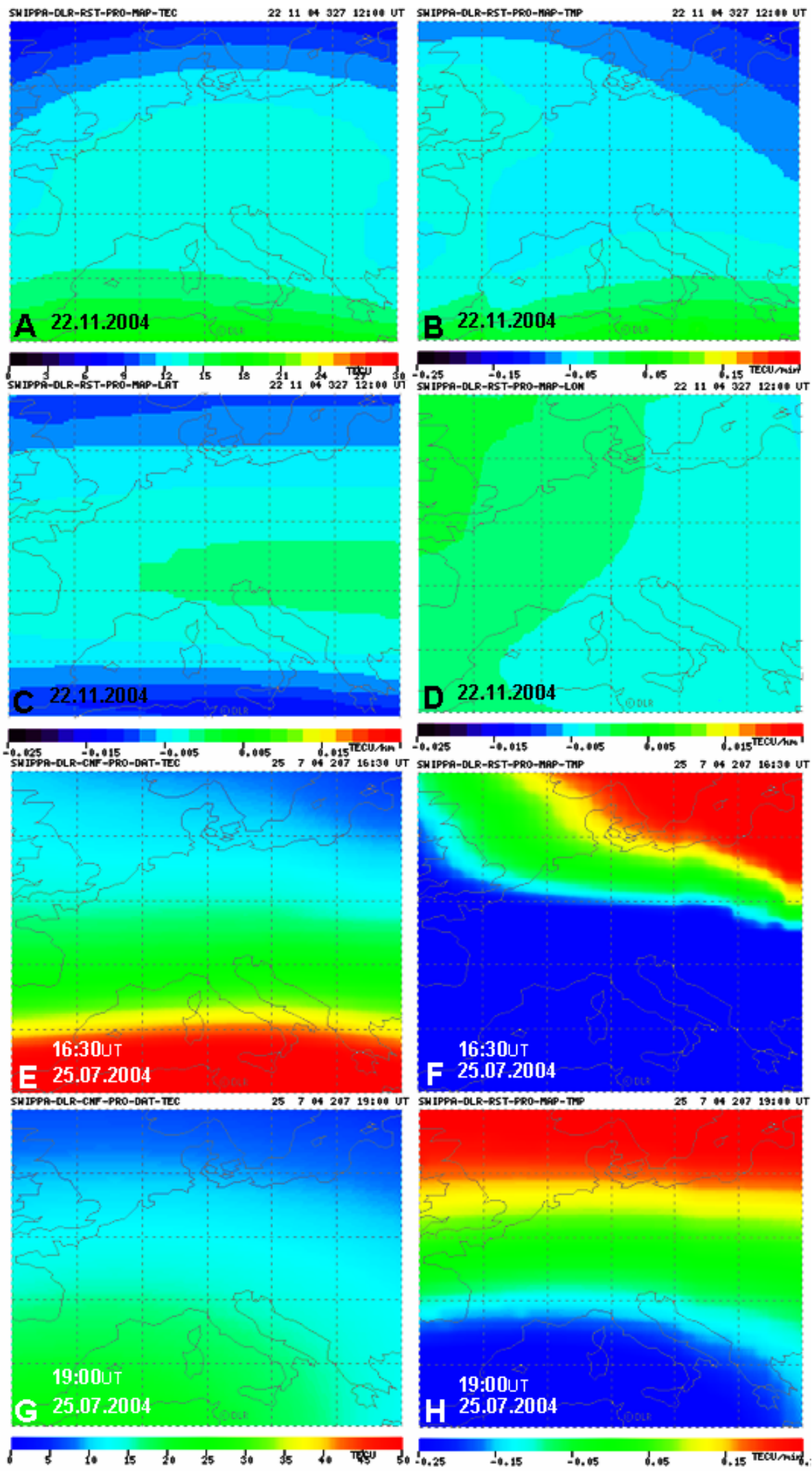

Fig. 6. SWIPPA products and services for the European area. Exemplary maps of the TEC (A), TEC temporal (B), latitudinal (C), and longitudinal (D) gradients at 12:00 UT on 22 November 2004. Maps of the TEC (E, G) and TEC temporal gradients (F, H) on 25 July 2004. 


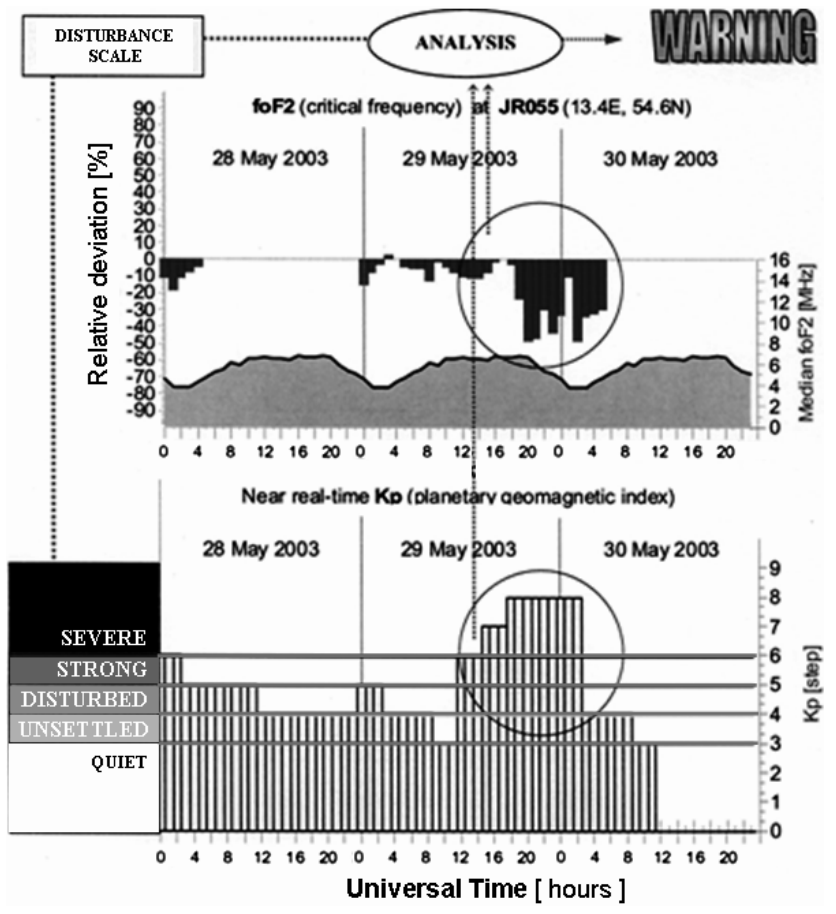

Fig. 7. Synthesis of warning geophysical messages based on nowcast/forecast information from ground- and space-based observations; an example based on the ionospheric storm events of 29-30 May 2003. The plot is based on real-time data which explains the existence of some gaps and possible inaccuracies in the estimation of the relative deviation; nevertheless, the storm effects are visible. The disturbance scale reflects preliminary estimations of the space-weather impact on positioning and will be further adjusted to different users/practices.

processed and solved satellites, and the decreased reference network reliability. Also demonstrated was the necessity of an operational nowcast and forecast service using realtime space weather observations. In relation to the abovementioned problems and the necessity of the space-weather monitoring service, the SWIPPA project was briefly described. This project utilises specific space-weather information in reference networks in order to help GNSS specialists to deliver a more reliable, precise and secure positioning service and to eventually reduce the operation, production, and other business costs. Development of forecast products and further improvement of the nowcast products, addressing the ionospheric space-weather effects on GNSS applications, are some of the objectives of the newly started project SWACI (Space Weather Application Centre - Ionosphere). Also, the Space Weather European Network (SWENET) has recently been established. SWENET aims at federating existing and newly created space weather services, assisting in the development of a common network, developing associated software infrastructure, providing support to service development activities, encouraging common development, undertaking public outreach activities, assessing user requirements, etc.
The operational space-weather service is currently being independently assessed and recommendations for further improvements are expected. However, in line with the increased sophistication of present-day technological systems, it is already clear that there will be a growing market for navigational and precise positioning applications in Europe, particularly when GALILEO becomes operational.

Acknowledgements. The SWIPPA project has been jointly supported by the German Aerospace Centre (DLR) and the European Space Agency (ESA) under contract ESTEC 16952/02/NL/LvH. The work was carried out within the framework of the ESA space weather applications pilot project and is part of the SWENET network.

The authors thank Allsat network+services GmbH, E.ON Ruhrgas AG, International GPS Service (IGS), NOAA Space Environment Center (SEC), German Federal Agency for Cartography and Geodesy (BKG), GeoForschungsZentrum Potsdam (GFZ), Adolf-Schmidt Geomagnetic Observatory Niemegk, Institute of Atmospheric Physics Kühlungsborn, and Troms $\emptyset$ Geophysical Observatory for kindly providing data needed for the SWIPPA project.

The SWACI project is funded by the German State Government of Mecklenburg-Vorpommern under grant V230-630-08-TIFA-334.

Gratefully acknowledged is the past and ongoing contribution of C. Becker, S. Schlueter, T. Noack, A. Wehrenpfennig, R. Favre, J. Mielich, H. J. Linthe, E. Daly, A. Glover, A. Hilgers, J. Rueffer, B. Huck, C. Daub, A. Rietdorf, J. Kunches, H. Luehr, J. Rubach, K. Behnke, A. Fischer, C. Hall, and O. Husoy.

Topical Editor T. Pulkkinen thanks I. Kutiev and another referee for their help in evaluating this paper.

\section{References}

Beniguel, Y.: Global Ionospheric Propagation Model (GIM): A propagation model for scintillations of transmitted signals. Radio Science, 37(3), 1032, doi:10.1029/2000RS002393, 2002.

Beniguel, Y., Adam, J. P., Jakowski, N., Noack, T., Schlueter, S., and Stankov, S. M.: Analysis of signal scintillations for the Scandinavian and equatorial regions. Presented at the International Beacon Satellite Symposium 2004, 18-22 October 2004, Trieste, Italy, 2004.

Boteler, D. H. and Jansen Van Beek, G.: 4 August 1972 revisited: A new look at the geomagnetic disturbance that caused the L4 cable system outage, Geoph. Res. Lett., 26, 577-580, 1999.

Costello, K. A.: Moving the Rice MSFM into a real-time forecast mode using solar wind driven forecast models, Ph.D. dissertation, Rice University, Houston TX, 1997.

Foerster, M. and Jakowski, N.: Geomagnetic Storm Effects on the Topside Ionosphere and Plasmasphere: A Compact Tutorial and New Results, Surveys in Geophysics, 21(1), 47-87, 2000.

Foster, J. C., Holt, J. M., Musgrove, R. G., and Evans, D. S.: Ionospheric Convection Associated with Discrete Levels of Particle Precipitation, Geophys. Res. Lett., 13, 656-659, 1986.

Harang, L.: Maximalwerte der Erdstromspannungen in der Nähe der Nordlichtzone während sehr intensiver erdmagnetischer Störungen, Gerl. Beitr. Geophys., 57, 310-316, 1941.

Jakowski, N.: TEC Monitoring by Using Satellite Positioning Systems, in: Modern Ionospheric Science, edited by: Kohl, H., 
Ruester, R., and Schlegel, K., EGS, Katlenburg-Lindau, 371390, 1996.

Jakowski, N., Sardon, E., Engler, E., Jungstand, A., and Klaehn, D.: Relationships between GPS-signal propagation errors and EISCAT observations, Ann. Geophys., 14, 1429-1436, 1996,

SRef-ID: 1432-0576/ag/1996-14-1429.

Jakowski, N., Hocke, K., Schlueter, S., and Heise, S.: Space Weather Effects Detected by GPS Based TEC Monitoring. Proc. ESA Workshop on Space Weather, 11-13 November 1998, ESTEC, WP-155, 241-243, 1998.

Jakowski, N., Schlueter, S., and Sardon, E.: Total Electron Content of the ionosphere during the geomagnetic storm on 10 January 1997, J. Atmos. Sol.-Terr. Phys., 61, 299-307, 1999.

Jakowski, N., Heise, S., Wehrenpfennig, A., Schlueter, S., and Reimer, R.: GPS/GLONASS-based TEC measurements as a contributor for space weather, J. Atm. Sol.-Terr. Phys., 64, 729735, 2002a.

Jakowski, N., Wehrenpfennig, A., Heise, S., and Kutiev, I.: Space weather effects on trans-ionospheric radio wave propagation on 6 April 2000. Acta Geodaetica et Geophysica Hungarica, 37(2-3), 213-220, 2002b.

Jakowski, N., Stankov, S. M., Klaehn, D., and Rueffer, J.: SWIPPA - Space Weather Impact on Precise Positioning Appplications of GNSS, in: Space Weather Applications Pilot Project (Proceedings of the ESA Space Weather Workshop, 16-18 December 2002, ESTEC, Noordwijk, The Netherlands), 2002c.

Jakowski, N., Wehrenpfennig, A., Heise, S., and Stankov, S. M.: Space weather effects in the ionosphere deduced from ground and space based GPS measurements, in: Space weather effects on communications and electric power distribution (Proc. NATO Advanced Research Workshop on Effects of Space Weather on Technology Infrastructure ESPRIT-2003, 25-29 March 2003, Rhodes, Greece), 2003a.

Jakowski, N., Stankov, S. M., Klaehn, D., and Rueffer, J.: Current developments in SWIPPA "Space Weather Impact on Precise Positioning Applications of GNSS", in: Developing a European Space Weather Service Network (Proc. European Space Agency Workshop on Space Weather, 3-5 November 2003, ESTEC, Noordwijk, The Netherlands, $2003 \mathrm{~b}$.

Jakowski, N., Stankov, S. M., Klaehn, D., Schlueter, S., Beniguel, Y., and Rueffer, J.: Operational service for monitoring and evaluating the space weather impact on precise positioning, Proc. European Navigation Conference GNSS 2004, 16-19 May 2004, Rotterdam, The Netherlands, 2004a.

Jakowski, N., Stankov, S. M., Klaehn, D., Schlueter, S., Noack, T., Beniguel, Y., Rueffer, J., Rietdorf, A., Huck, B., and Daub, C.: Adverse space weather effects on precise positioning - case studies, in: Satellite Navigation User Equipment Technologies, ESA-WPP239, Proc. Second ESA Workshop on Satellite Navigation User Equipment Technologies - NAVITEC 2004, 8-10 December 2004, ESTEC, The Netherlands, 758-765, 2004b.
Kappenman, J. G.: Geomagnetic storms and their impact on power systems, IEEE Power Engineering Review, 16, 5, 1996.

Koskinen, H., Eliasson, L., Holback, B., Andersson, L., Eriksson, A., Maelkki, A., Norberg, O., Pulkkinen, T., Viljanen, A., Wahlund, J. E., and Wu, J. G.: Space weather and interactions with spacecraft, SPEE Final Report, FMI, Helsinki, 1999.

Langley, R. B.: GPS, the ionosphere, and the solar maximum, GPS World, 11(7), 44-49, 2000.

Makela, J. J., Kelley, M. C., Sojka, J. J., Pi, X. J., and Mannucci, A. J.: GPS normalization and preliminary modelling results of total electron content during a midlatitude space weather event, Radio Science, 36(2), 351-361, 2001.

Moulsley, T. J. and Vilar, E.: Experimental and theoretical statistics of microwave amplitude scintillations on satellite downlinks. IEEE Transactions on Antennas and Propagation, AP30(6), 1099-1106, 1982.

Oksanen, P. J.: Estimated individual annual cosmic radiation doses for flight crews. Aviation Space and Environmental Medicine, 69(7), 621-625, 1998.

Pirjola, R., Viljanen, A., Amm, O., and Pulkkinen, A.: Power and pipelines (ground systems), Proc. ESA Workshop on Space Weather, WPP-155, 45-52, 1999.

Skone, S. and Shrestha, S. M.: Limitations in DGPS positioning accuracies at low latitudes during solar maximum, Geophys. Res. Lett., 29(10), 1439, doi:10.1029/2001GL013854, 2002.

Stankov, S. M.: Ionosphere-plasmasphere system behaviour at disturbed and extreme magnetic conditions. OSTC Final Scientific Report, Royal Meteorological Institute of Belgium, Brussels, Belgium, 2002.

Stankov, S. M., Kutiev, I., Jakowski, N., and Wehrenpfennig, A.: A new method for total electron content forecasting using global positioning system measurements, Proc. ESTEC Workshop on Space Weather, 17-19 Dec. 2001, Noordwijk, The Netherlands, 169-172, 2001.

Stankov, S. M. and Jakowski, N.: GPS-based monitoring of the European and Polar ionosphere, Pres. First European Space Weather Workshop ESWW-2004, 29 November-3 December 2004, ESTEC, Noordwijk, The Netherlands, 2004.

Stankov, S. M., Jakowski, N. Wilken, V., and Tsybulya, K.: Generation and propagation of ionospheric disturbances studied by ground and space based GPS techniques, Proc. Ionospheric Effects Symposium (IES), 3-5 May 2005, Alexandria VA USA, Paper No. A064/9B2, 2005.

Walter, T., Datta-Barua, S., Blanch, J., and Enge, P.: The effects of large ionospheric gradients on single frequency airborne smoothing filters for WAAS and LAAS, Proc. ION NTM 2004, 26-28 January 2004, San Diego, CA, 2004. 